\title{
The kinematic preshaping of triggered self-adaptive linkage-driven robotic fingers
}

\begin{abstract}
L. Birglen
Ecole Polytechnique of Montreal, Department of Mechanical Engineering, Montreal, QC, H3T 1J4, Canada

Received: 28 February 2010 - Revised: 29 June 2010 - Accepted: 19 August 2010 - Published: 8 February 2011

Abstract. In this paper, the issue of the kinematic - as opposed to dynamic - preshaping of self-adaptive robotic fingers driven by linkages is discussed. A method to obtain designs of these fingers capable of various behaviours during their closing motions is presented. The method is based on using triggered passive elements in carefully selected joints of the finger and the selection or optimization of geometric parameters to obtain particular kinematic relationships between the motions of the phalanges. This method is very general and can be applied to any self-adaptive robotic finger in order to obtain many different types of closing motions. Examples given in this paper are focusing on two different preshaping motions, the first one aims at allowing pinch grasps while the second mimics a human finger. The fundamental aim of this paper is to show that various preshapings of self-adaptive fingers are possible, not just one, and to give two step-by-step examples.
\end{abstract}

This paper was presented at the IFToMM/ASME International Workshop on Underactuated Grasping (UG2010), 19 August 2010, Montréal, Canada.

\section{Introduction}

\subsection{Self-adaptive fingers}

In the past few years, a significant increase in the development of innovative technologies has tried to address the lack of commercial success of complex robotic hands. Significant efforts have been made to find designs simple enough to be easily built and controlled (Bicchi, 2000), particularly in human prosthetics. A special emphasis has been placed on the reduction of the number of degrees of freedom (DOF), thereby decreasing the number of required actuators. In particular, the SSL hand (Akin et al., 2002), the DIES-DIEM hand (Biagiotti et al., 2001) and the Cassino finger (Figliolini and Ceccarelli, 2002) have followed this path. On the other hand, a rapidly growing number of prototypes involve a smaller number of actuators without decreasing the number of DOF by taking advantages of self-adaptive, a.k.a. underactuated, mechanisms. These prototypes are usually either driven by tendons (Hirose and Umetani, 1978; Crisman et al., 1996; Massa et al., 2002; Higashimori et al., 2005a) or by linkages (Bekey et al., 1999; Ulrich, 1988; Laliberté and Gosselin, 1998; Kennedy, 2001; Ceccarelli et al.,

Correspondence to: L. Birglen

(lionel.birglen@polymtl.ca) 2006b). This approach leads to the automatic/mechanical adaptation of the robotic finger to the shape of the object seized. A classic example of a self-adaptive two-DOF finger driven by linkages and its closing sequence is illustrated in Fig. 1.

Self-adaptive mechanisms offer tremendous possibilities and have been known for decades but they have been kept in the shadows of the exponentially growing capability of digital architectures. These mechanical systems have been superseded by their electronic counterpart which provided better, more accurate, cheaper and faster means of performing computing tasks (Gosselin, 2006). However, the confinement of mechanical systems to the execution of motion under the control of a higher-level controller has to be considered as a design choice rather than an obligation. In this paper:

- the kinematic preshaping of self-adaptive fingers is defined,

- two examples are given: pinch and anthropomorphic with the two-phalanx class $S$ finger,

- geometric parameters to achieve these preshapings are either selected or optimized,

- limitations and extensions of the proposed methodology are discussed. 


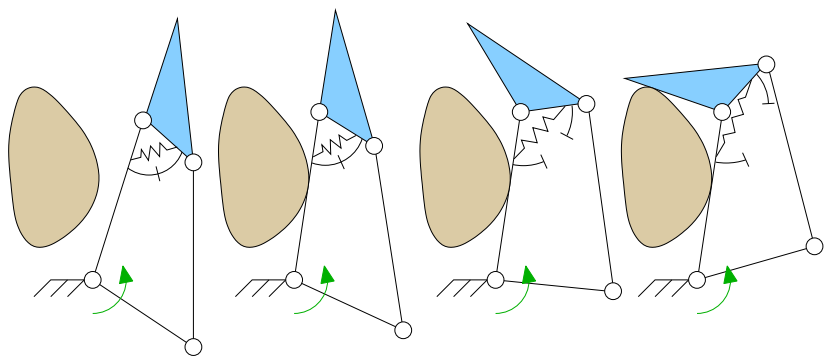

Figure 1. Closing sequence of a two-DOF self-adaptive finger.

\section{Kinematics of triggered self-adaptive fingers}

\subsection{Introduction}

The essence of self-adaptive fingers is twofold: a transmission linkage used to transmit actuation to the phalanges, and passive elements used to constrain the finger. In this paper, it is assumed that the finger is driven by a single actuator and unless otherwise specified the latter is located between the ground and the transmission linkage. Passive elements, as illustrated in Fig. 1, are key to prevent any undesired motions of the finger since the latter has $n$-DOF while having as few as one actuator. It is the aim of this paper to present how, by carefully selecting the type and location of these passive elements, several very interesting behaviours can be achieved. Many different types of passive elements may be considered (Birglen, 2009). Each of them applies a constraint (i.e. a torque/force) to a selected joint in the linkage. Springs and triggered springs are by far the most popular choice to achieve this goal. Using a triggered element in one of the joints of a self-adaptive finger, one locks this joint when no external constraint if applied to the mechanism, i.e. when no contact has yet occurred. Therefore, with a $n$-phalanx self-adaptive finger when $n-1$ triggered elements are used, one obtains a single DOF linkage. If a reasonable number (e.g. less than 6) of binary/ternary links is considered, the only possible one-DOF linkages (Birglen, 2009) are the fourbar, Watt's and Stephenson's linkages, illustrated in Fig. 2.

Hence, when no external contact has yet occurred, the self-adaptive finger considered behaves as one of these three linkages. Therefore, if triggered elements are used, a purely kinematic relationship exists between the angles of the phalanges $\theta_{i}$ and actuation, i.e. one has

$g_{i}\left(\theta_{i}, \theta_{\mathrm{a}}\right)=0 \quad$ with $\quad i=1, \ldots, n$

with a $n$-phalanx finger where $\theta_{\mathrm{a}}$ is the angle associated with actuation. A trivial case occurs when the triggered element(s) is (are) located between the phalanges $j$ and $j-1$ or between the ground and the proximal phalanx. Both cases respectively yields $\theta_{j}=K$ or $\theta_{1}=L$ where $K$ and $L$ are constants. If a triggered element is located elsewhere in the mechanism, i.e. in the transmission linkage, the functions $g_{i}$ can be
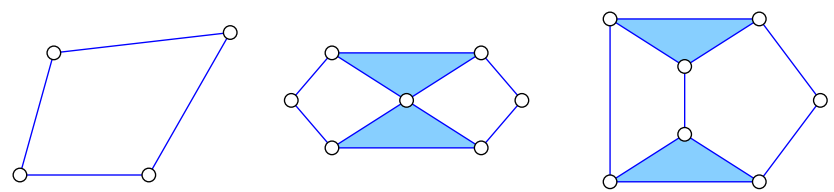

Figure 2. Four-bar, Watt's and Stephenson's one-DOF linkages.

obtained by writing the loop-closure equations of the linkage and a relationship $h\left(\theta_{1}, \ldots, \theta_{n}\right)$ can be obtained. With Watt's and Stephenson's linkages, this might be challenging as these equations are highly nonlinear and closed-form solutions have not been reported in the literature to the best of the author's knowledge (except in few cases with simplified geometries). Nevertheless, numerical values can be obtained. On the other hand, if the finger is reduced to a four-bar linkage, a closed-form solution exists. The position analysis of a four-bar linkage is actually a well-known textbook problem (Norton, 1992; McCarthy, 2000) and the relationships between the angles of the driven/driving cranks and the coupler can be obtained. Even if only the four-bar case is considered, a wide range of behaviours can be obtained as will be shown.

\subsection{Kinematics of the class $S$}

As an example, let us consider the linkage-driven twophalanx class $S$ finger (Birglen, 2009). A class $S$ finger with $n$ phalanges is constituted by a single loop linkage with $n+3$ joints. The phalanges and the ground to which they are attached are chosen as consecutive links of the mechanism and connected by revolute joints. Previously, only the case where this finger presented the particularity of having a ground link reduced to a single point was usually considered (cf. Fig. 1). This choice considerably simplifies mechanical design and the finger based on this simplification has been extensively investigated (Birglen et al., 2008). However, the more general case where two distinct joints constitute the ground link of the mechanism allows for much more freedom. It is worth mentioning that this class can be easily extended to an arbitrary number of phalanges. Indeed, the definition of this class is that the transmission mechanism is a simple planar motion generator (e.g. a RRR or RPR chain).

Triggered elements placed in any joint(s) of such a threephalanx finger to reduce its DOF during the closing motion results in a four-bar linkage, as illustrated in Fig. 3. In this figure, the "lock" symbol has been used to indicate which joint holds the triggered element and the resulting four-bar linkage is highlighted in dashed lines. The kinematic reduction of the finger to a four-bar is true for any finger of this class when an appropriate number of triggered elements is used. A maximum number of two passive elements can be located in the joints of the transmission linkage since the latter is constituted by only three joints and the actuated joint 

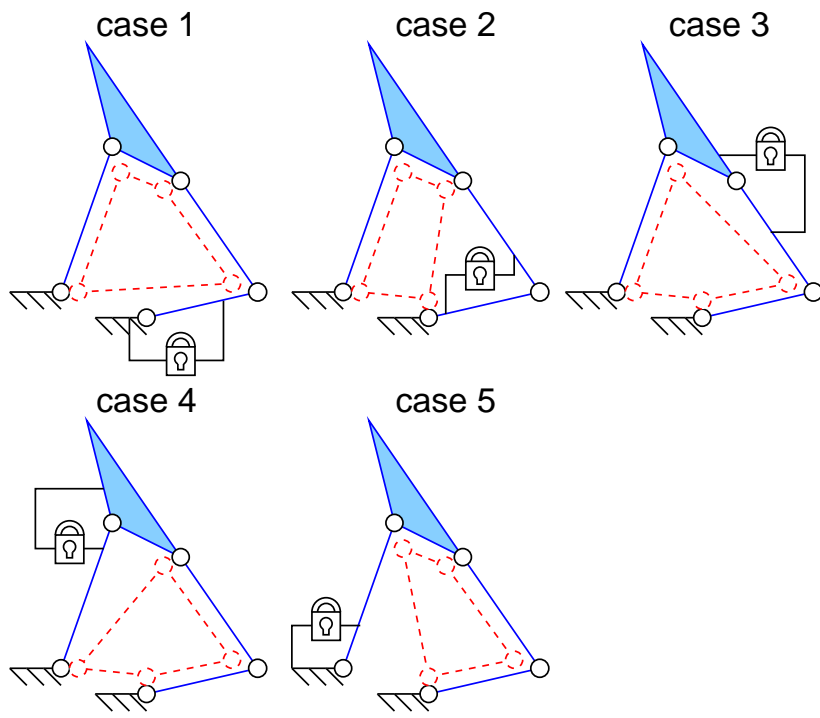

Figure 3. Kinematic reductions of the two-phalanx class S finger with one triggered element.

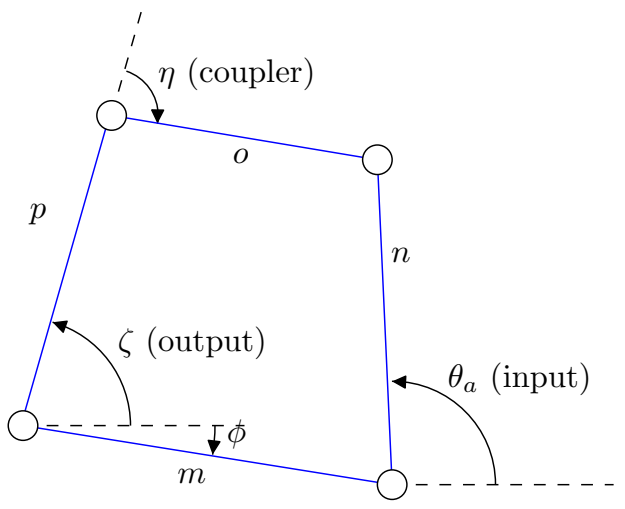

Figure 4. Modeling of a four-bar linkage.

is not a proper choice (although in certain cases it can hold a passive element, e.g. with compliant fingers). Therefore, if more passive elements are required they are necessarily located between the phalanges. Yet, the final one-DOF linkage remains a four-bar.

Let us consider the kinematic modeling of a general fourbar linkage illustrated in Fig. 4. Using the loop-closure equations, the relationships between the output/coupler, output/input and coupler/input angles are obtained. One has respectively:

$$
\begin{array}{r}
p^{2}+o^{2}+m^{2}-n^{2}+2 o p \cos \eta-2 m p \cos (\zeta-\phi) \\
-2 m o \cos (\phi-\zeta-\eta)=0, \\
m^{2}+n^{2}+p^{2}-o^{2}+2 m n \cos \left(\phi-\theta_{\mathrm{a}}\right) \\
-2 m p \cos (\phi-\zeta)-2 n p \cos \left(\theta_{\mathrm{a}}-\zeta\right)=0, \\
m^{2}+n^{2}-o^{2}-p^{2}+2 m n \cos \left(\theta_{\mathrm{a}}-\phi\right)-2 o p \cos \eta=0 .
\end{array}
$$

With the two-phalanx finger and disregarding the trivial cases
Table 1. Associations of geometric parameters.

\begin{tabular}{cccccccc}
\hline & $m$ & $n$ & $o$ & $p$ & $\phi$ & $\zeta$ & $\eta$ \\
\hline Case 1 & $r$ & $b$ & $c$ & $l_{1}$ & $\alpha+\beta$ & $\theta_{1}$ & $\theta_{2}-\psi$ \\
Case 2 & $d$ & $s$ & $c$ & $l_{1}$ & $\alpha$ & $\theta_{1}$ & $\theta_{2}-\psi$ \\
Case 3 & $d$ & $a$ & $t$ & $l_{1}$ & $\alpha$ & $\theta_{1}$ & $\theta_{2}-\psi-\gamma$ \\
\hline
\end{tabular}

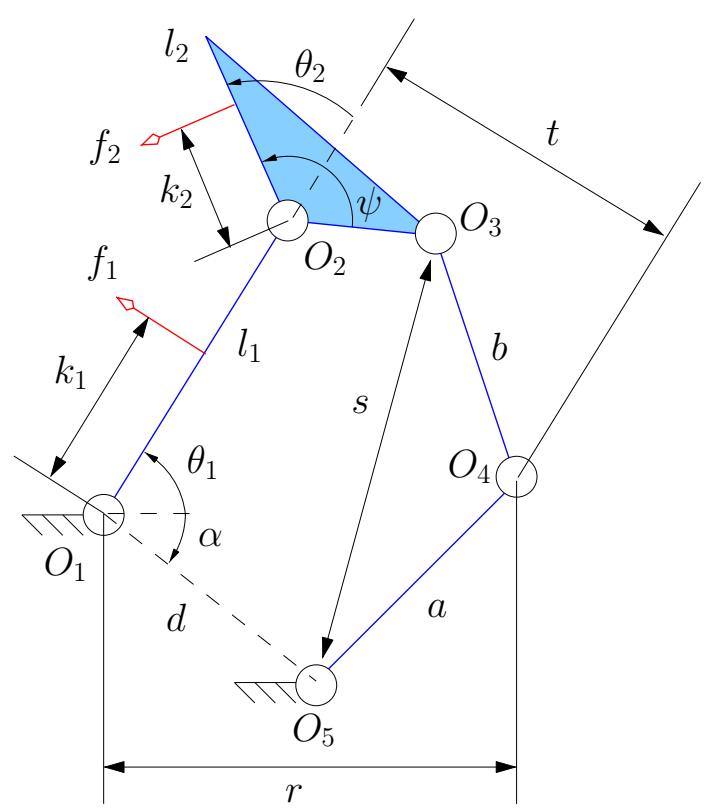

Figure 5. Details of the class $S$ two-phalanx finger.

4 and 5, each location for the passive element yields a different association between the geometric parameters of the four-bar with these of the finger (illustrated in Fig. 5) as described in Table 1.

In this table, $r$ is the distance between $O_{1}$ and $O_{4}, s$ is the distance between $O_{3}$ and $O_{5}$ and $t$ is the distance between $O_{2}$ and $O_{4}$. Each one of these distances is kept constant in its respective case. The angles $\beta$ and $\gamma$ are defined by

$\beta=\arccos \left(\frac{r^{2}+d^{2}-a^{2}}{2 r d}\right)$ and $\gamma=\arccos \left(\frac{c^{2}+t^{2}-b^{2}}{2 c t}\right)$

Note that in case 1, actuation is assumed to be located in $O_{4}$.

Equation (2) is very important since it describes the relationship between the output phalanx angles of the mechanism directly, i.e. without $\theta_{\mathrm{a}}$. By carefully choosing the geometric parameters of the fingers and therefore the coefficients of this equation, it is possible to approximate several very interesting relationships between $\theta_{1}$ and $\theta_{2}$ for the finger, as will be shown. 


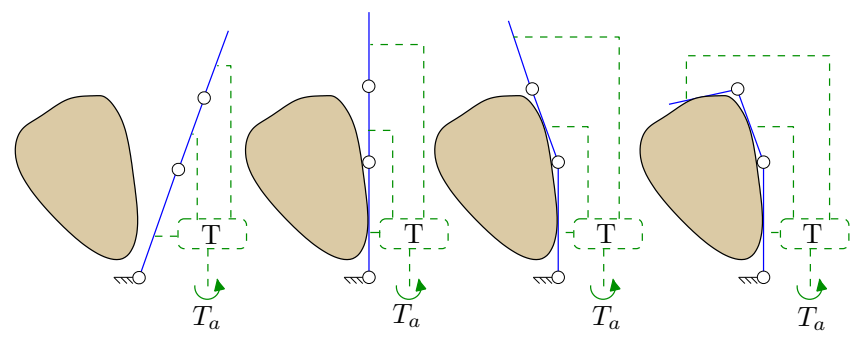

Figure 6. Closing sequence of a three-phalanx finger keeping its phalanges aligned.

\section{Kinematic preshaping}

\subsection{Introduction and straight line preshaping}

First, let us briefly recall the definition of preshaping: the preshaping of a finger - artificial or not - is defined as the set of geometric configurations this finger undertakes during the pregrasping phase, when no contact has yet occurred (Zhiwei et al., 2002; Supuk et al., 2005). With self-adaptive fingers, this issue has been overlooked in the past because of the limited set of available kinematic architectures known to be able to achieve self-adaption. Indeed, to the best of the author's knowledge, most prototypes of self-adaptive robotic fingers have springs located between their phalanges (or a kinematically equivalent design) in order for the latter to stay aligned during the pregrasping phase (as illustrated in Figs. 1 and 6). This preshaping can be done with any architectures of selfadaptive robotic finger since by definition, the phalanges are consecutive links of the mechanism and therefore, it is possible to locate spring(s) and mechanical limit(s) between them.

Recently, the Robotics Laboratory of Hiroshima University has proposed a method to optimize the preshaping of one self-adaptive robotic finger originally proposed in Hirose and Umetani (1978). This method was dealing with dynamic preshaping (Higashimori et al., 2005b), a particular aspect of the preshaping issue based on a dynamic analysis. Indeed, the proposed finger was subjected to very high accelerations (the authors claim to approach $100 \mathrm{~g}$ ). With such high accelerations, dynamics becomes a critical issue and a specific dynamic preshaping, optimized to increase the probability for the finger to make contact with a spherical object using all its phalanges, was proposed based on a refined dynamic modeling. However, this method requires a thorough knowledge of the dynamics of the system which yields a significant burden to the designer and is unnecessarily complicated when low accelerations are considered. Furthermore, although this method is very accurate, results obtained are difficult to extend to other designs, for instance fingers based on linkages as in this paper.

On the other hand, kinematic preshaping as proposed here is defined as the preshaping of a self-adaptive finger from a quasi-static perspective, i.e. when dynamics is neglected in favor of kinematics. This approach does not require any knowledge of the dynamic of the system. Triggered elements are key to this method since they prevent motions under the assumption that dynamic forces are small. Continuous elements on the other hand are much more sensitive to these forces. Furthermore, triggered passive elements allow to consider simpler one-DOF linkages as discussed in Sect. 2 and illustrated in Fig. 2 instead of the complete mechanism. For instance, the preshaping of two-phalanx fingers such as the one described in Fig. 5 is the set of both phalanx joint angles when it is reduced to one of the linkages in Fig. 2. Disregarding again trivial cases where the triggered element is located in one of the phalanx joints, the preshaping can be described by a set of two equations, namely

$\left\{\begin{array}{l}\theta_{1}=g_{1}\left(\theta_{\mathrm{a}}\right) \\ \theta_{2}=g_{2}\left(\theta_{\mathrm{a}}\right)\end{array}\right.$.

Therefore, providing that $g_{1}$ and $g_{2}$ are invertible (at least locally), the preshaping can be described by a single equation, namely

$g_{1}^{-1}\left(\theta_{1}\right)=g_{2}^{-1}\left(\theta_{2}\right) \Leftrightarrow h\left(\theta_{1}, \theta_{2}\right)=0$.

When the finger can be reduced to a four-bar linkage, Eq. (7) simply becomes Eq. (2). Following the same method, the preshaping of an $n$-phalanx finger can be described by a set of $n-1$ equations. This set of equations usually cannot match a particular desired preshaping except in some simple cases (e.g. in Sect. 3.2). However, the difference between the sets of equations describing the actual and desired preshaping can be characterized by a performance index and an optimization procedure can be used to maximize this index.

\subsection{Pinch preshaping}

An interesting feature of a few self-adaptive robotic fingers proposed in the literature (Gosselin and Laliberté, 1996; Bartholet, 1992; Bégoc et al., 2004) is the ability to maintain their distal phalanx perpendicular to the palm during their closing sequence. This kinematic property allows pinch grasps as illustrated in Fig. 7. This is very useful when the finger is seizing objects with linear edges. It should be noted that the shape adaptation capability must be kept to perform enveloping grasps if required (upper part of Fig. 7). The preshaping equation characteristic of a finger capable of pinch grasps is

$\sum_{i=1}^{n} \theta_{i}=\frac{\pi}{2}$

In this case, this preshaping can be described by a single (and simple) equation. To achieve this feature, prototypes have been developped using various solutions such as a complex cam-linkage mechanism that can be disengaged (Bartholet, 1992), an additional linkage (Gosselin and Laliberté, 1996), or more recently, a combination of two interconnected 


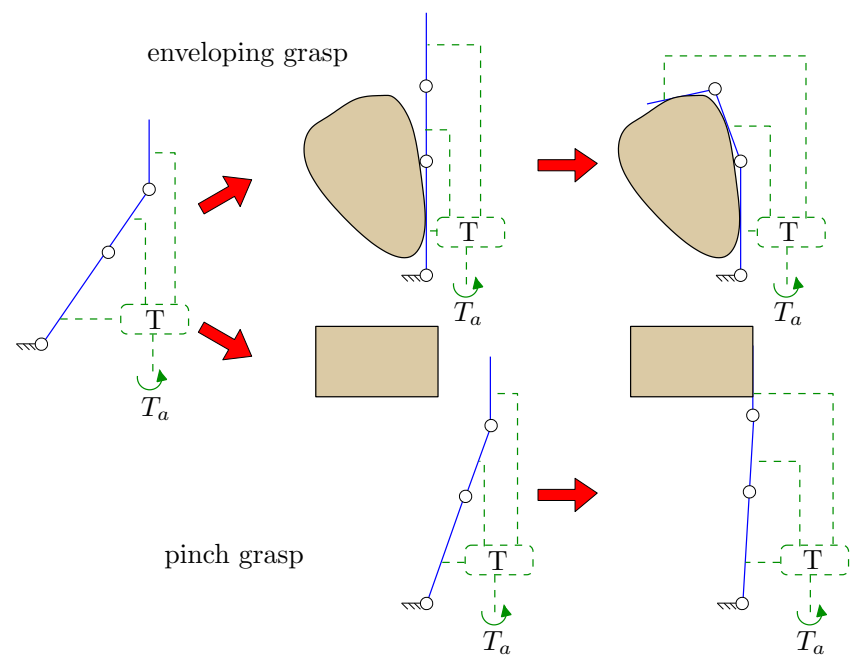

Figure 7. Two possible closing sequences of a three-phalanx finger designed to allow pinch grasps.

struts with non-reversible valves dubbed the air parallelogram (Bégoc et al., 2004). These solutions have been reported to work well but they all require an addition to the transmission linkage used for self-adaptation. However, if one carefully uses triggered elements in certain joint(s) of the mechanism, this behaviour can be closely approached without requiring any addition.

It is well known that a parallelogram linkage maintains its coupler link parallel to the ground. This property can be used in the design of self-adaptive robotic fingers with pinch preshaping. For instance, let us consider the mechanical architectures presented in Fig. 3. Non-trivial cases $(1-3)$ can be very easily kinematically reduced to a parallelogram if simple geometric conditions on the lengths of the links are satisfied. Namely, in case 1 they are $b=l_{1}$ and $r=c$. In case 2 , they become $c=d$ and $s=l_{1}$. Finally, in case 3 one has $a=l_{1}$ and $t=d$. If these conditions are satisfied, Eq. (2) becomes

$\theta_{1}+\theta_{2}=\phi-\eta+\theta_{2}$

This equation is valid with any of the three cases considered and it is trivial to show that the right hand side of the previous equation is constant, i.e. $\theta_{2}$ is canceled in all cases by $\eta$ and only the design parameters are left. Therefore, it is possible to choose these parameters to exactly match Eq. (8). Hence, for the distal phalanx of a two-phalanx class $S$ finger to maintain a constant orientation with respect to the ground, one simply has to place a triggered element in one joint of the transmission linkage, adjust the lengths of the latter in order for the resulting one-DOF mechanism to be a parallelogram and choose the remaining geometric parameters to ensure that Eq. (9) matches Eq. (8). The method is illustrated in Fig. 8, the green circular arrows indicate possible actuation location. With these designs, the distal phalanx will remain

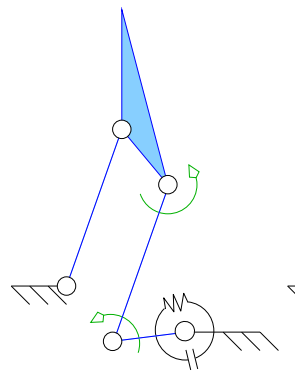

case 1

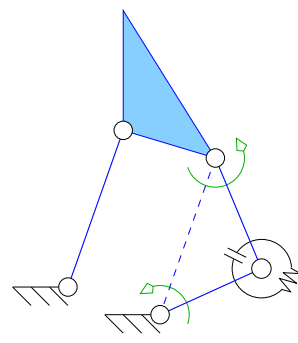

case 2

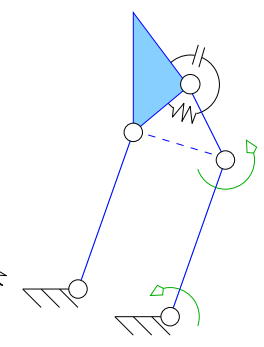

case 3
Figure 8. Designs of the two-phalanx class $S$ architecture allowing pinch preshaping.

perpendicular to the palm until a contact occurs. When this contact is established, the actuation torque will overcome the preloading of the triggered element and initiate the closing of the other phalanx. Hence, pinch preshaping is achieved without any additional mechanisms.

Nevertheless, if the contact occurs with the distal phalanx during this pregrasping phase, the resulting grasp stability must be studied to determine if the linear contact can be maintained. Indeed, with a contact on only one phalanx, i.e. one constraint, a two-DOF finger without additional mechanisms is not necessarily in static equilibrium as discussed in Birglen and Gosselin (2004). If this is not the case, contact with the whole line will be lost and a sliding motion of the finger in contact with only one vertex of the object will occur. This contact situation can lead to either a stable grasp or ejection (Birglen and Gosselin, 2006b). It should be noted that the parallelogram linkages proposed in Fig. 8 are known to achieve very poorly with respect to grasp stability. However, this is not an issue here because the parallelogram shape is only maintained during the preshaping phase and pinch grasps. It is automatically disengaged when a contact does not occur with the distal phalanx.

Let us consider the case 2 of Fig. 8 . The equilibrium equation is defined as a set of contact locations on the distal phalanx leading to a static equilibrium of the finger with no contact on the proximal phalanx, i.e. the set of $k_{2}$ such as $f_{1}=0$ (cf. Fig 5). Note that this situation corresponds to the desired pinch grasp. The equilibrium equation of the finger discussed here is

$e\left(\theta_{1}, \theta_{2}\right)=k_{2}=h_{4} \cos \theta_{2}$

where $h_{4}$ is the signed distance between $O_{2}$ and the intersection of lines $\left(\mathrm{O}_{1} \mathrm{O}_{2}\right)$ and $\left(\mathrm{O}_{3} \mathrm{O}_{4}\right)$. The equilibrium equation value $e$ is a particular location of the contact force on the distal phalanx (not the value of the force but its location) and a function of $h_{4}$ which is itself a complex function of $\theta_{1}$ and $\theta_{2}$. The equilibrium equation defines a set of contact locations (a surface) in the grasp-state space $\left(\theta_{1}, \theta_{2}, k_{2}\right)$. Note that its value can be smaller than zero or greater than $l_{2}$, i.e. outside the physical limits of the phalanx. The equilibrium equation 


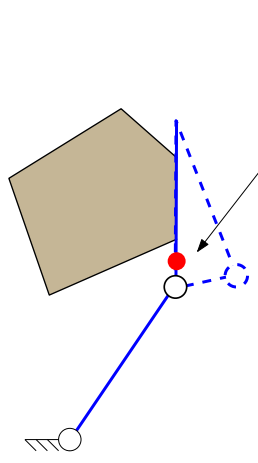

Unstable

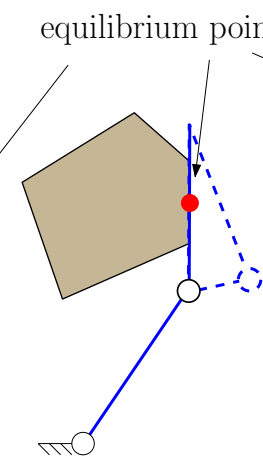

stable

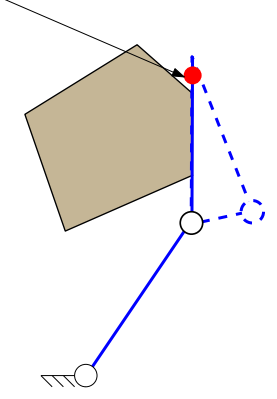

Unstable
Figure 9. Stability of a planar pinch grasp depending on the equilibrium location.

is critical with respect to the grasp stability of a linear contact. It has also been demonstrated in Birglen and Gosselin (2006b) that a linear contact with the distal phalanx of a twophalanx self-adaptive finger is stable if and only if the location of the equilibrium position is located between both vertices of the line (cf. Fig. 9). However, it should be noted that if this condition cannot be met, ejection will not necessarily occur. Indeed, the grasp can still be stable if $e\left(\theta_{1}, \theta_{2}\right)<l_{2}$ but with a contact maintained only with one of the edges of the object.

Therefore, the stability of a linear grasp cannot be guaranteed in all cases, since the object cannot be assumed to have a linear contact with the entire distal phalanx. However, in most common cases, the linear contact is long enough to exceed the physical limit of the distal phalanx, i.e. the rightmost case of Fig. 9 can be disregarded, and a compromise can be found. This compromise lies between the avoidance of ejection, favored by a small value of the equilibrium location, and the ability to perform pinch grasp, favored by a large value of the equilibrium location. This compromise can be part of the optimization procedure characterized by an index $I_{e}^{*}$ :

$I_{e}^{*}=\left\{\begin{array}{l}0 \text { if } \max (e)_{W}>l_{2} \\ \min \left(e / l_{2}\right)_{W^{*}} \text { otherwise }\end{array}\right.$

where $W^{*}$ is the set of phalanx angles during the preshaping, i.e. the locus described by $\theta_{1}+\theta_{2}=\pi / 2$. This performance index is to be maximized. It ensures that the equilibrium value $e$ is the closest possible to $l_{2}$ over the workspace $W^{*}$ (its minimal value is maximized) without being greater than $l_{2}$ (the score drops to zero).

If the lengths of the proximal and distal phalanges are respectively chosen as 1 and $l_{1} / \sqrt{2}=0.707$. The first step of the optimization is to find out an initial estimate of the geometric parameters. This initial choice can be done very easily by choosing the parameters to form a parallelogram. Then a numerical optimization toolbox can be used to further refine the design. Taking into account the constraints mentioned

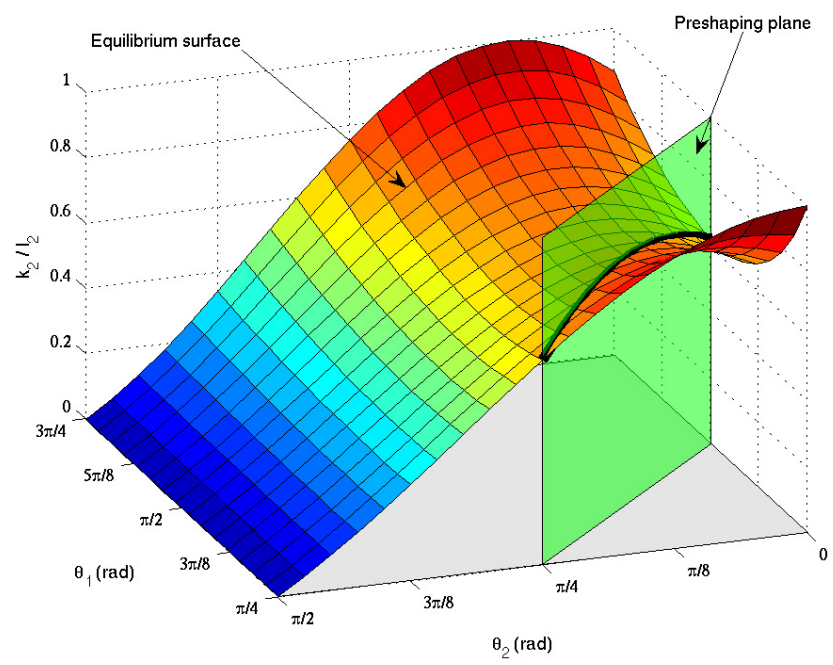

Figure 10. Equilibrium surface of the optimal design of two-phalanx finger with pinch preshaping.

Table 2. Two-phalanx finger optimal geometric parameters.

\begin{tabular}{cccccc}
\hline$\psi$ & $a$ & $b$ & $c$ & $d$ & $\alpha$ \\
\hline $95^{\circ}$ & 0.84 & 1.32 & 0.39 & 0.39 & $-5^{\circ}$ \\
\hline
\end{tabular}

above, one can reduce the original set of 8 geometric parameters to only four: $\alpha, a, b$ and $c$ in case 2 (since $\psi=\pi / 2-\alpha$ and $d=c$ ) to speed up calculations. An example of the result of such an optimization procedure is presented in Table 2 corresponding to the equilibrium locus illustrated in Fig. 10. Note that the set of angles $\theta_{1}$ and $\theta_{2}$ during the preshaping phase, denoted $W^{*}$, corresponds to a plane in the grasp-state space and the associated values of the equilibrium locations - maximized through the index $I_{e}^{*}$ - are outlined in black in Fig. 10. The maximal value of the equilibrium equation over the workspace $W$ does not exceed 1 . Hence, no ejection occurs and the finger is always stable. The minimal value of the equilibrium equation in the preshaping phase $W^{*}$ is $62.1 \%$ of $l_{2}$. This means that any linear contact with one vertex below $0.621 l_{2}$ and the other beyond the limit the phalanx is stable. This behaviour has been verified with a virtual prototype using a dynamic simulation package.

The procedure leading to this two-phalanx design can be adapted to a finger with three phalanges by taking into account the grasp stability and the expressions of the contact forces of three-phalanx fingers (Birglen and Gosselin, 2006a). Furthermore, it should be emphasized that this method is actually not limited to one class of kinematic architectures (the class $S$ in this example). In order to maintain the desired orientation of the distal phalanx, one can use cascaded parallelograms as illustrated in Fig. 11. In this figure, three architectures from Birglen (2009) are illustrated 

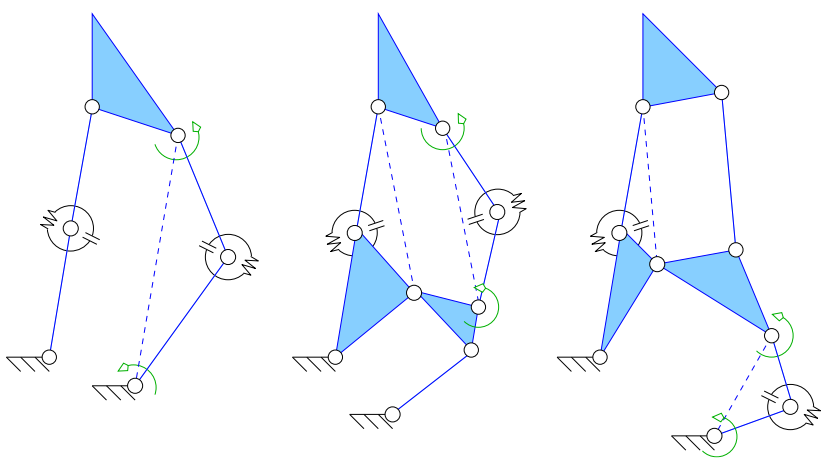

Figure 11. Three-phalanx self-adaptive fingers with pinch preshaping.

where two triggered elements are used to maintain the orientation of the distal phalanx during the closing preshaping. The green circular arrows indicate possible actuation location, but it should be noted that only one location has to be chosen, i.e. all three fingers have three DOF but can be driven by a single actuator.

\subsection{Anthropomorphic preshaping}

When designing prosthetic fingers, two approaches are possible. Either attention is paid to the closing motion of the prosthesis to mimic a human finger, i.e. an anthropomorphic preshaping (Figliolini and Ceccarelli, 2002; Ceccarelli et al., 2006b,a), or to the forces generated by the phalanges (de Visser and Herder, 2000). Since the latter approach does not require a particular closing motion, it has lead to the introduction of self-adaptive fingers in prosthetics (de Visser and Herder, 2000; Lotti and Vassura, 2002; Carrozza et al., 2004). However, it is possible to design an artificial finger that has an anthropomorphic closing motion while keeping the self-adaptation property as also very recently proposed in Wu et al. (2009). In Figliolini and Ceccarelli (2002), it is illustrated that in the absence of obstacles, the closing motion of a human finger exhibits approximately linear relationships between the rotations of the individual phalanges. Namely, one has

$\theta_{\mathrm{i}}=m_{1}\left(\theta_{\mathrm{p}}-\pi / 2\right) \quad$ and $\quad \theta_{\mathrm{d}}=m_{2}\left(\theta_{\mathrm{p}}-\pi / 2\right)$,

where $\left(\theta_{\mathrm{p}}, \theta_{\mathrm{i}}, \theta_{\mathrm{d}}\right)$ are respectively the proximal, intermediate and distal relative angles between the phalanges. Numerical values reported in Figliolini and Ceccarelli (2002) are used in this paper, i.e. $\left(m_{1}, m_{2}\right)=(3.96,2.37)$ with $90^{\circ} \leq \theta_{\mathrm{p}} \leq 197^{\circ}$. Using again the two-phalanx finger illustrated in Fig. 5, the mechanism is reduced to a four-bar linkage by locking one joint to approximate the motion described by Eq. (12). If the resulting four-bar linkage is used to drive the last two phalanges of a prosthetic finger, i.e. $\theta_{1}=\theta_{i}$ and $\theta_{2}=\theta_{\mathrm{d}}$, the preshaping equation is

$\theta_{2}=m \theta_{1} \quad$ with $\quad m=m_{2} / m_{1}$.

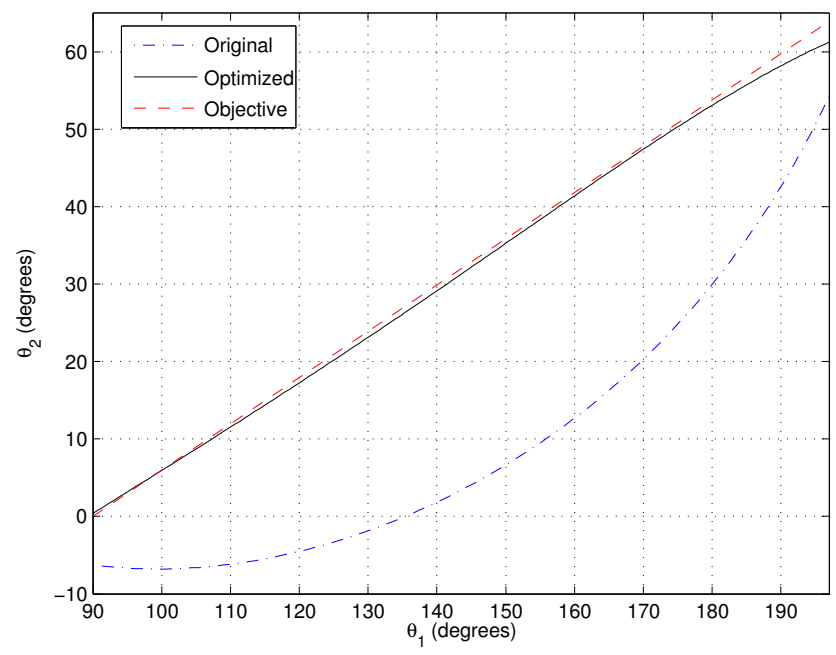

Figure 12. Comparison of preshaping motions.

Table 3. Two-phalanx finger second optimal geometric parameters.

\begin{tabular}{cccccc}
\hline$\psi$ & $a$ & $b$ & $c$ & $d$ & $\alpha$ \\
\hline $119^{\circ}$ & 0.94 & 0.81 & 0.29 & 0.20 & $-149^{\circ}$ \\
\hline
\end{tabular}

It is obvious that Eq. (2) is highly nonlinear and cannot usually be reduced into Eq. (13). However, a performance index measuring the closeness of the desired preshaping to the actual motion can be used, e.g.

$I_{\mathrm{a}}=\frac{1}{W^{*}} \int_{W^{*}} \frac{1}{1+\left(\frac{f^{*}\left(\theta_{1}\right)}{m \theta_{1}}-1\right)^{2}} d \theta_{1}$

where $f^{*}\left(\theta_{1}\right)$ is the solution of Eq. (2) for $\theta_{2}$. Again, in the case of a four-bar linkage it is interesting to note that a closed-form expression of $f^{*}\left(\theta_{1}\right)$ exists (McCarthy, 2000) although its expression is impractical. The workspace of the finger is defined here as $W=\left\{90<\theta_{1}<197^{\circ}, 0<\theta_{2}<64^{\circ}\right\}$ to match the workspace proposed in Figliolini and Ceccarelli (2002) and $I_{\mathrm{a}}$ is therefore evaluated for $W^{*} \equiv 90<\theta_{1}<$ $197^{\circ}$. If the joint locked during the preshaping is $O_{4}$ (case 2 in Fig. 8) the same optimization procedure discussed in the previous section yields the results presented in Table 3 . Again, these parameters have been obtained with $l_{1}=1$ and $l_{2}=l_{1} / \sqrt{2}=0.707$. This time, the initial estimate has been chosen geometrically to have approximately the desired initial and final position, disregarding intermediate behaviour. A comparison of the initial, optimal and desired preshaping motions is illustrated in Fig. 12.

The equilibrium surface associated with this optimal set of geometric parameters is illustrated in Fig. 13. Equation (2) for any value of $k_{2}$ defines in this grasp-state space a preshaping surface illustrated in Fig. 13. Note that ideally, this surface should be a plane as the objective of the optimization 


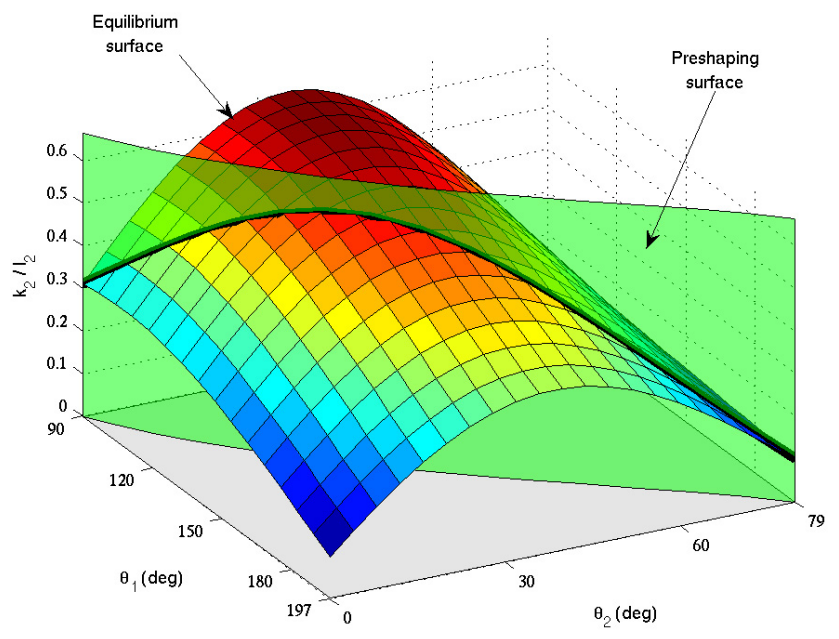

Figure 13. Equilibrium surface of the optimal design of twophalanx finger with anthropomorphic preshaping.

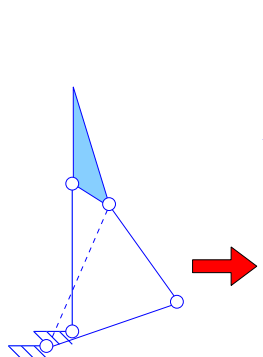

(a)

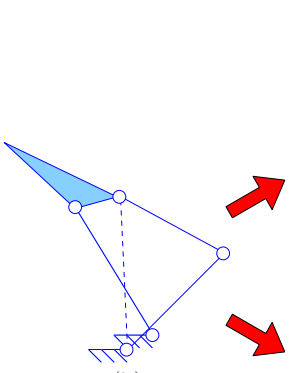

(b)

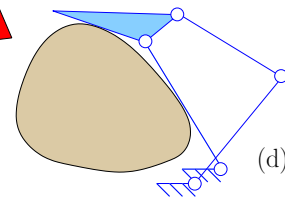

Figure 14. Possible closing motions of the optimized finger.

procedure was to approach Eq. (13). The finger built with these parameters and with a triggered passive element in $O_{4}$ will have a preshaping close to a human finger while keeping the self-adaptation property as illustrated in Fig. 14.

\section{Conclusions}

The issue of the kinematic preshaping of linkage-driven selfadaptive finger was discussed in this paper. A general method was proposed to achieve various preshapings based on using triggered passive elements. These elements reduce the number of DOF of self-adaptive fingers to one and allow to study the motion undergone by these fingers when no contact has occurred. Based on basic kinematic properties of four-bar linkages, designs of self-adaptive fingers were proposed that allow pinch grasps without requiring any additional mechanisms. The method was subsequently used in another example aiming at mimicking the closing motion of a human finger. These two examples of preshapings were demonstrated
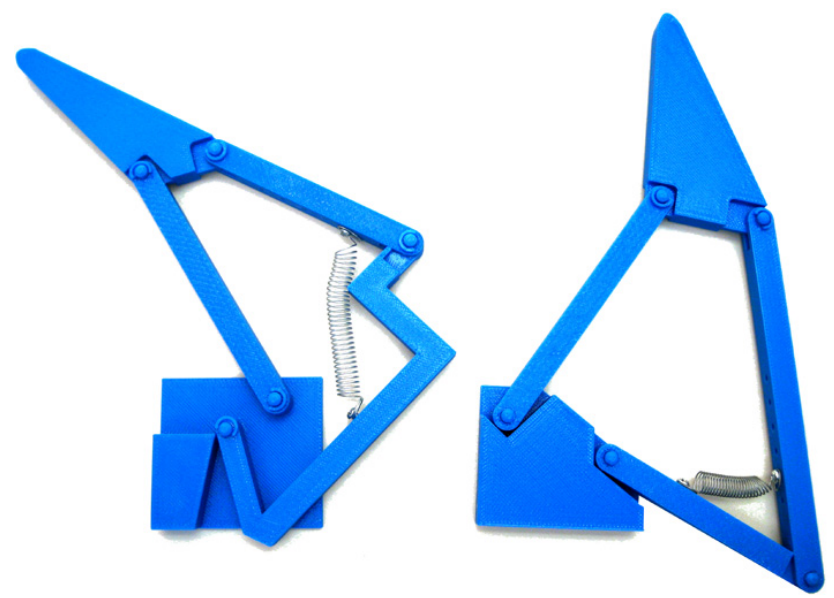

Figure 15. Prototypes of self-adaptive fingers with the discussed preshapings.

experimentally during the 2010 IFToMM/ASME International Workshop on Underactuated Grasping using fused deposition models illustrated in Fig. 15. Finally, only two different types of preshaping were discussed in this paper and with only one simple class of self-adaptive fingers. However, it is probable that any kind of motion is achievable using other architectures of self-adaptive fingers and that the presented preshapings can also be achieved by most of these other architectures.

Acknowledgements. The financial support of the Natural Sciences and Engineering Research Council of Canada (NSERC) and the Fonds québécois de la recherche sur la nature et les technologies (FQRNT) is gratefully acknowledged.

Edited by: J. L. Herder

Reviewed by: two anonymous referees

\section{References}

Akin, D. L., Carignan, C. R., and Foster, A. W.: Development of a Four-Fingered Dexterous Robot End Effector For Space Operations, Proceedings of the 2002 IEEE International Conference on Robotics and Automation, Washington, DC, USA, 2302-2308, 2002.

Bartholet, S. J.: Reconfigurable End Effector, US Patent No. 5108140, 1992.

Bégoc, V., Krut, S., Dombre, E., Durand, C., and Pierrot, F.: Mechanical Design of a New Pneumatically Driven Underactuated Hand, Proceedings of the IEEE International Conference on Robotics and Automation, 927-933, Roma, Italy, 2004.

Bekey, G. A., Tomovic, R., and Zeljkovic, I.: Control Architecture for the Belgrade/USC Hand in Dextrous Robot Hands, SpringerVerlag, New-York, 1999.

Biagiotti, L., Melchiorri, C., and Vassura, G.: Control of a Robotic Gripper for Grasping Objects in No-Gravity Conditions, Proceedings of the 2001 IEEE International Conference on Robotics and Automation, 1427-1432, Seoul, Korea, 2001. 
Bicchi, A.: Hands for Dexterous Manipulation and Powerful Grasping: A Difficult Road Towards Simplicity, IEEE T. Robotic. Autom., 16, 652-662, 2000.

Birglen, L.: The Synthesis of Linkage-Driven Self-Adaptive Fingers, ASME Journal of Mechanisms and Robotics, 1, 809-816, 2009.

Birglen, L. and Gosselin, C.: Kinetostatic Analysis of Underactuated Fingers, IEEE T. Robotic. Autom., 20, 211-221, 2004.

Birglen, L. and Gosselin, C.: Geometric Design of Three-Phalanx Underactuated Fingers, J. Mech. Des.-T. ASME, 128, 356-364, 2006a.

Birglen, L. and Gosselin, C.: Grasp-State Plane Analysis of TwoPhalanx Underactuated Fingers, Mech. Mach. Theory, 41, 807822, 2006b.

Birglen, L., Laliberté, T., and Gosselin, C.: Underactuated Robotic Hands, Springer, New-York, 2008

Carrozza, M. C., Suppo, C., Sebastiani, F., Massa, B., Vecchi, F., Lazzarini, R., Cutkosky, M. R., and Dario, P.: The SPRING Hand: Development of a Self-Adaptive Prosthesis for Restoring Natural Grasping, Auton. Robot., 16, 125-141, 2004.

Ceccarelli, M., Rodriguez, N. E. N., and Carbone, G.: Design and tests of a three finger hand with 1-DOF articulated fingers, Robotica, 24, 183-196, 2006a.

Ceccarelli, M., Tavolieri, C., and Lu, Z.: Design Considerations for Underactuated Grasp with a one D.O.F. Anthropomorphic Finger Mechanism, 2006 IEEE/RSJ International Conference on Intelligent Robots and Systems, 1611-1616, 2006b.

Crisman, J. D., Kanojia, C., and Zeid, I.: Graspar: A Flexible, Easily Controllable Robotic Hand, IEEE Robot. Autom. Mag., 3238, 1996.

de Visser, H. and Herder, J. L.: Force Directed Design of a Voluntary Closing Hand Prosthesis, Proceedings of the 2000 ASME Design Engineering Technical Conferences, Baltimore, MD, USA, 1-8, 2000.

Figliolini, G. and Ceccarelli, M.: A Novel Articulated Mechanism Mimicking the Motion of Index Fingers, Robotica, 20, 13-22, 2002.

Gosselin, C.: Adaptive Robotic Mechanical Systems: A Design Paradigm, J. Mech. Des.-T. ASME, 128, 192-198, 2006.

Gosselin, C. and Laliberté, T.: Underactuated mechanical finger with return actuation, US Patent No. 5762390, 1996.
Higashimori, M., Kaneko, M., Namiki, A., and Ishikawa, M.: Design of the 100G Capturing Robot Based on Dynamic Preshaping, Int. Jo. Robot. Res., 24, 743-753, 2005a.

Higashimori, M., Nishio, S., and Kaneko, M.: Dynamic Preshaping Based Optimum Design for High Speed Capturing Robots, Proceedings of the 2005 IEEE/RSJ International Conference on Intelligent Robots and Systems, Edmonton, Canada, 913-918, 2005b.

Hirose, S. and Umetani, Y.: The Development of Soft Gripper for the Versatile Robot Hand, Mech. Mach. Theory, 13, 351-358, 1978.

Kennedy, B.: Three-Fingered Robot Hand With Self-Adjusting Grip, Nasa Tech Briefs, 25, 59, 2001.

Laliberté, T. and Gosselin, C.: Simulation and Design of Underactuated Mechanical Hands, Mech. Mach. Theory, 33, 39-57, 1998.

Lotti, F. and Vassura, G.: A Novel Approach to Mechanical Design of Articulated Fingers for Robotic Hands, Proceedings of the 2002 IEEE/RSJ International Conference on Intelligent Robots and System, Vol. 2, 1687-1692, 2002.

Massa, B., Roccella, S., Carrozza, M. C., and Dario, P.: Design and Development of an Underactuated Prosthetic Hand, Proceedings of the 2002 IEEE International Conference on Robotics and Automation, Washington, DC, USA, 3374-3379, 2002.

McCarthy, J. M.: Geometric Design of Linkages, Springer-Verlag, 2000.

Norton, R. L.: Design of Machinery, McGraw-Hill, Inc., 1992.

Supuk, T., Kodek, T., and Bajd, T.: Estimation of Hand Preshaping During Human Grasping, Med. Eng. Phys., 27, 790-797, 2005.

Ulrich, N. T.: Methods and Apparatus for Mechanically Intelligent Grasping, US Patent No. 4957320, 1988.

Wu, L., Carbone, G., and Ceccarelli, M.: Designing an underactuated mechanism for a 1 active DOF finger operation, Mech. Mach. Theory, 44, 336-348, doi:10.1016/j.mechmachtheory. 2008.03.011, 2009.

Zhiwei, L., Ito, T., Sugimoto, N., Odashima, T., and Hosoe, S.: Virtual impedance control for preshaping of a robot hand, Proceedings of the 41st SICE Annual Conference, Osaka, Japan, 20582059, 2002. 